OPEN

SUBJECT AREAS:

BIOMECHANICS

PHYSIOLOGY

BIOMATERIALS

Received

5 February 2014

Accepted

21 May 2014

Published

12 June 2014

Correspondence and requests for materials should be addressed to

T.K. (tkleinteich@ zoologie.uni-kiel.de)

\section{Tongue adhesion in the horned frog Ceratophrys sp.}

\author{
Thomas Kleinteich \& Stanislav N. Gorb
}

Christian-Albrechts-Universität Kiel, Functional Morphology and Biomechanics, Am Botanischen Garten 1-9, 24118 Kiel, Germany.

Frogs are well-known to capture elusive prey with their protrusible and adhesive tongues. However, the adhesive performance of frog tongues and the mechanism of the contact formation with the prey item remain unknown. Here we measured for the first time adhesive forces and tongue contact areas in living individuals of a horned frog (Ceratophrys sp.) against glass. We found that Ceratophrys sp. generates adhesive forces well beyond its own body weight. Surprisingly, we found that the tongues adhered stronger in feeding trials in which the coverage of the tongue contact area with mucus was relatively low. Thus, besides the presence of mucus, other features of the frog tongue (surface profile, material properties) are important to generate sufficient adhesive forces. Overall, the experimental data shows that frog tongues can be best compared to pressure sensitive adhesives (PSAs) that are of common technical use as adhesive tapes or labels.



he evolution of a tongue for prey capture and transport on land is considered an important innovation of terrestrial vertebrates (i.e. Tetrapoda) ${ }^{1}$. Within tetrapods, several lineages evolved sticky tongues that use adhesion to catch pull prey into the mouth. In chameleons (Sauropsida: Squamata: Chamaeleonidae), many frogs (Lissamphibia: Anura), and some plethodontid salamanders (Lissamphibia: Caudata: Plethodontidae), these adhesive tongues can be fired ballistically onto distant targets ${ }^{2-12}$. While the morphology, kinematics, and neuromuscular control of these highly protrusible tongues have been studied in detail over several dec$\operatorname{ades}^{7,13-19}$, the actual adhesive mechanism that allows tongues to stick to prey surfaces received much less attention (but see $\mathrm{Herrel}^{20}$ for an account on chameleon tongue adhesion).

Several species of frogs with protrusible tongues are known to consume very large prey compared to their own body dimensions (see Branch ${ }^{21}$, Chávez et al. ${ }^{22}$, Duellman and Trueb ${ }^{23}$, Figueiredo-de-Andrade et al. ${ }^{24}$, or McLeod $^{25}$ for examples). Among these are frogs of the Ceratophryidae, a group comprising twelve species in the three genera Ceratophrys, Chacophrys, and Lepidobatrachus (AmphibiaWeb, 2013). Characteristic features of the Ceratophryidae are their large heads compared to their body size and their hyper-ossified skulls ${ }^{26-28}$. Frogs of the genus Ceratophrys are known to be sit-and-wait predators that feed on a vast variety of different prey species, including lizards, snakes, rodents, frogs, annelids, crabs, spiders, and insects ${ }^{29}$. Chávez et al. ${ }^{22}$ reported on an individual of Ceratophrys cornuta preying on another frog that was almost two thirds of its own body size (measured by snout-vent-length).

The ability to catch large and very distinctive prey in frogs of the Ceratophryidae puts special demands on the adhesive performance of their protrusible tongues. The attachment to the prey must at least be strong enough to prevent the prey from escaping before it is grasped by the jaws - depending on the size of the prey, it will even be lifted off the ground and actively pulled into the mouth of the frog ${ }^{30}$. The timeframes at which the frog tongue is in contact with the prey are extremely short and usually fall into the range of 10 to $60 \mathrm{~ms}^{7,8,31-33}$. Frog tongue surfaces are covered by mucus ${ }^{34,35}$ and thus can be considered a wet adhesive system. However, the relationship between the force applied to the surface, contact area, and adhesion remains unclear. Also the role of mucus in the adhesive mechanism is unresolved.

Here, we measured the in vivo adhesive performance of the tongue in frogs of the genus Ceratophrys (Ceratophryidae) by motivating the animals to fire their tongues towards microscope glass slides that were connected to a force transducer and behind whose we presented a visual stimulus. With this experimental setup, we were also able to study the tongue prints that were left on the glass slides in detail and to directly measure the contact area of the tongue. The aims of this study were: (1) to measure the adhesive forces that frog tongues can generate against a smooth surface (glass), (2) to determine the adhesive strength of frog tongues on glass (i.e. adhesive force over contact area), (3) to examine how adhesive strength depends on (a) the contact pressure during the tongue impact, (b) the duration of the feeding event, and (c) the utilization of mucus. 


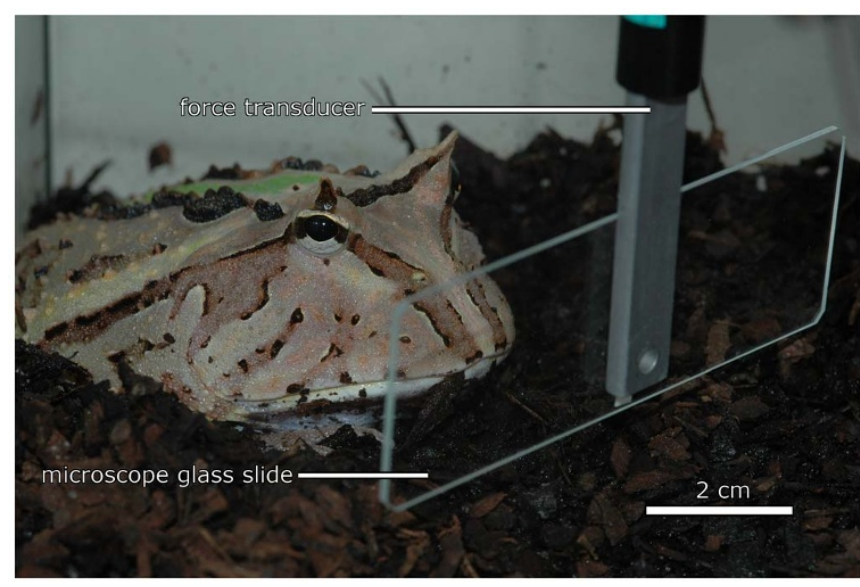

Figure 1 Experimental setup. A force transducer with an attached glass slide was mounted approximately $2 \mathrm{~cm}$ in front of Ceratophrys cranwelli $\times$ cornuta kept into the terrarium. Because the animals are pronounced sitand-wait predators, they did not react during the building up of the experiment until we presented them food items (crickets) behind the force transducer. Photograph ${ }^{\circledR}$ T. Kleinteich.

\section{Results}

Force measurements. Our experimental setup (Fig. 1) allowed to record force over time relationships while the tongues of the frogs were in contact with microscope glass slides, attached to a force transducer (Fig. 2). The force over time relationships for our force measurement experiment show a short and very distinct peak for the tongue impact (Fig. 2G) which was on average $2.92 \pm 1.58$ the body weight of the frogs. The highest impact force per body weight ratio we measured was 6.49 in a juvenile frog that weighed $12.7 \mathrm{~g}$ and had a tongue impact force of $806 \mathrm{mN}$ (see Supplementary table 1 for force measurement data for each feeding trial). Impact force relative to the tongue contact area, i.e. contact pressure, was on average $6.07 \mathrm{kPa}$. The highest contact pressure, which we recorded for the impact of a frog tongue on a glass slide was $28.6 \mathrm{kPa}$. The tongue impact duration was very short; on average $39.1 \pm 19.6 \mathrm{~ms}$.

Immediately after the impact force peak, the force over time graphs showed a clear adhesive force peak in opposite direction. Depending on the movements of the frog and its tongue after this first peak, we observed a notable drop in the pulling force before a prolonged phase in which we constantly measured pulling forces that were, however, notably smaller than at the initial peak for the adhesive force (Fig. 2G). In some cases, during the transition between the initial peak of the adhesive curve and the prolonged pulling phase, we measured no pulling forces, indicating, that the frogs were either pushing their tongues or their heads towards the force transducer (Fig. 2D, G) before they started to pull their tongues off. The pulling forces at the first and highest peak were on average $1.45 \pm 0.66$ times the body weight of the frogs. The highest adhesive force to body mass ratio, which we measured was 3.4 in a juvenile frog which at a body mass of $13.7 \mathrm{~g}$ pulled with $456 \mathrm{mN}$ during one experimental trial. We found adhesive strength (i.e. the ratio of the adhesive force over the tongue contact area) for frog tongues on glass to be on average $3.01 \pm 2.53 \mathrm{kPa}$ with a maximum recorded adhesive strength of $17.7 \mathrm{kPa}$. The duration of the adhesive phase was much longer compared to the impact phase and lasted on average 1,132.5 $\pm 747.2 \mathrm{~ms}$.

The impulse for the pulling phase in our experiment, i.e. the force which was applied to the microscope glass slide over the total duration of the pulling phase, was on average $0.19 \pm 0.13$ Ns. For an experimental trial in which the $63 \mathrm{~mm}$ SVL adult frog pulled with a peak adhesive force of $613 \mathrm{mN}$ and in which the adhesive phase lasted 1,845 ms, we measured the maximal impulse herein with 0.77 Ns.
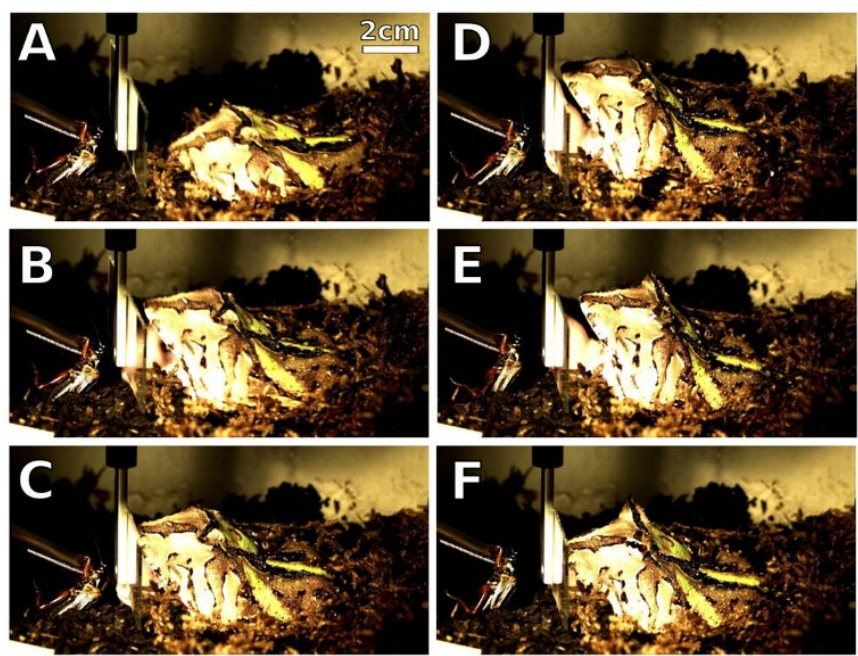

G

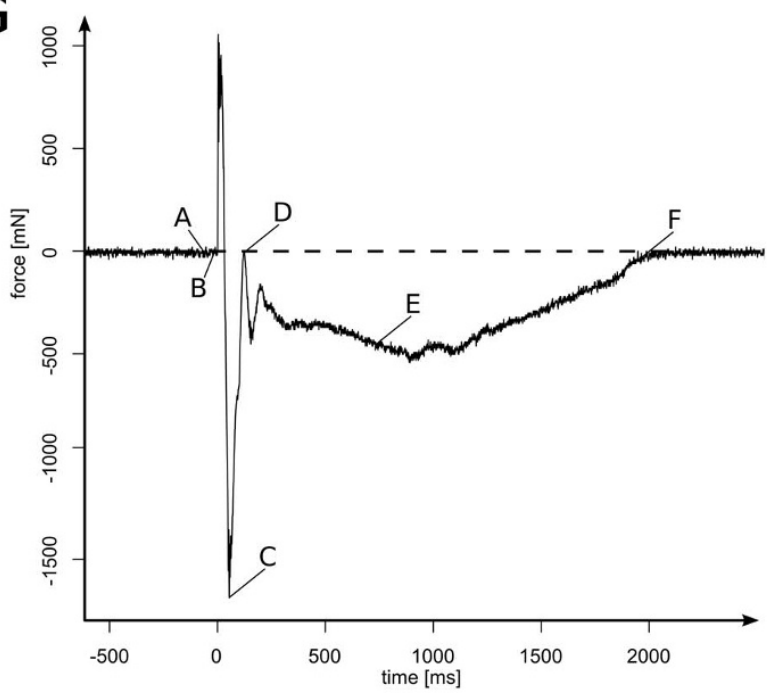

Figure $2 \mid$ Representative experimental trial. (A-F): still frames from a high speed video sequence showing how the frog adheres to and detaches its tongue from the glass slide; $(G)$ : force over time curve, highlighting the specific force values in time that refer to the still frames in $(\mathrm{A}-\mathrm{F})$. Positive force values relate to pushing forces (i.e. tongue impact); negative force values relate to pulling forces (i.e. adhesion). The video was captured at 500 frames per second and the entire video sequence is available as

Supplementary video file 1. (A): before the impact onto the glass slide, the frog lunges towards the cricket. (B): contact initiation. The mouth of the frog is widely open and the dorsal surface of the tongue gets in contact with the glass slide. (C): the moment at which the measured adhesive force is maximal. By lunging towards the target, the frog is in close proximity to the glass slide with the tongue attached to it. (D): depending on the movements of the frog and its tongue, we observed some variation in the force after the initial peak of adhesive force. Here, the pulling force drops to almost zero, indicating that the frog is not pulling its tongue at this special moment. However, the video sequence shows that the tongue still is in full contact with the glass slide. (E): pulling phase. The frog is moving backwards and we measured pulling forces again. However, during this phase, pulling is notably weaker than at the initial peak of the adhesion curve. (F): tongue contact release. The tongue is not in contact with the glass slide anymore. Photographs ${ }^{\circledR}$ T. Kleinteich.

The analysis of the tongue prints after each experimental trial revealed strong variation in the amount of mucus that remained on the glass slides after the tongue was detached (Fig. 3). Relative mucus coverage of the tongue prints ranged from $1 \%$ of the tongue 

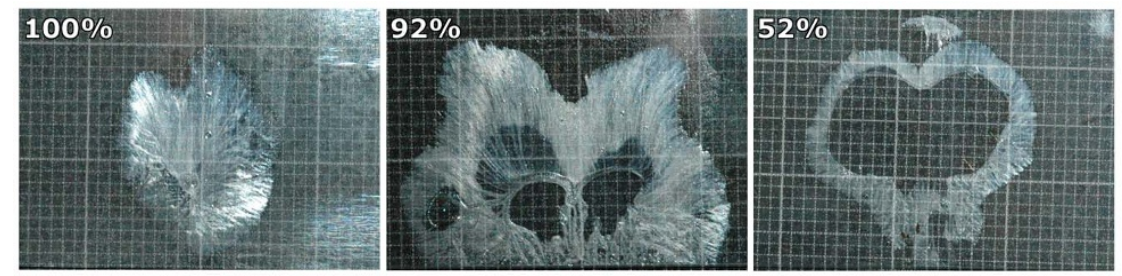

Figure 3 Tongue prints on microscope glass slides; the scale paper in the background is $1 \mathrm{~mm} \times 1 \mathbf{m m}$. The values in the upper left corner depict the relative mucus coverage of the overall contact area. We observed a high degree of variation in the shape of frog tongue prints and relative mucus coverage (ranging from $1 \%$ to $100 \%$ ).

contact area (i.e. only very thin margins of mucus were detectable) to $100 \%$.

Statistical analysis. Table 1 shows the ANOVA table for the results of four multivariate linear regression analyses that we performed with (1) the adhesive force, (2) the adhesive strength, (3) the relative mucus coverage, and (4) the impulse for the pulling phase as dependent variables; Table 2 shows the estimated slopes for each independent variable in our regression models and $p$-values as indication if the slopes were significantly different from 0 (i.e. no relationship).

Adhesive force. The force of the impact, the duration of the tongue impact, the duration of the pulling phase, the size of the contact area, and the individual specimen IDs all explained significant portions of the observed variation in the adhesive force (Table 1). The duration of the impact of the tongue was significantly negatively related to the measured adhesive forces, i.e. shorter impact times resulted in stronger adhesion (Table 2). Our multivariate linear regression model was overall highly significant ( $\mathrm{p}=1.10 \mathrm{E}-13 ; \mathrm{F}=15.71$ on 9 and 70 degrees of freedom; adjusted $r^{2}=0.63$ ) and the residuals of the regression for the adhesive force were found to be normally distributed $(\mathrm{p}=0.88 ; \mathrm{W}=0.99)$. The four specimens tested herein differed significantly in the adhesive force they were able to maintain with their tongues (ANOVA, $\mathrm{p}=1.2 \mathrm{E}-15 ; \mathrm{F}=40.1$ on 3 and 76 degrees of freedom; Fig. 4).

Adhesive strength. The strength of the adhesion, i.e. adhesive force per area was significantly related to the contact pressure, the timespan during which the tongue was pulled towards the mouth of the frog (i.e. pulling duration), the degree of mucus coverage in the contact area, and the individual for which adhesive strength was measured (Table 1). Higher impact stress resulted in a stronger adhesive strength. Further, adhesive strength significantly increased with decreased durations of the pulling phase and with a decrease of the relative mucus coverage (Table 2). The regression model with adhesive strength as dependent variable herein was significant $(\mathrm{p}=$ 4.92E-09; $\mathrm{F}=9.77$ on 8 and 71 degrees of freedom; adjusted $\mathrm{r}^{2}=$ 0.47). A Shapiro-Wilks test revealed that the residual values for adhesive strength were normally distributed ( $\mathrm{p}=0.76$; $\mathrm{W}=0.99$ ). A one-way ANOVA of adhesive strength over specimen ID found no significant difference in the average adhesive strength of the animals ( $p=0.91 ; F=0.18$ on 3 and 76 degrees of freedom; Fig. 4 ) - the influence of the specimen ID on the adhesive strength as predicted by the multivariate regression analysis appears to be rather an effect of differences in adhesion duration between individuals (two-way ANOVA with adhesive strength as dependant on ID and adhesion duration; $\mathrm{p}=0.01, \mathrm{~F}=3.94$ on 3 and 76 degrees of freedom).

Relative mucus coverage. The variation in the degree of mucus coverage of the contact area observed herein was significantly related to adhesive strength, the duration of the tongue impact onto the glass slide, the duration of the pulling phase, the specimen ID, and the consecutive trial number during one series of force measurements on a given day (Table 1). We found that the strength of the adhesion was negatively correlated with the area covered by mucus (Table 2), i.e. in feeding trials after which we found larger patches of mucus on the glass slides, the adhesive strength was significantly lower. Further, a longer duration of the pulling phase relates to a significantly higher relative mucus coverage while in consecutive experimental trials, the mucus coverage decreased (Table 2). The multivariate linear regression model used herein shows overall a highly significant depen-

Table 1 ANOVA table for four different multivariate linear regression models

Response variable: adhesive force $F_{\text {adh }}$

\begin{tabular}{lccc}
\hline Independent variable & DF & F value & p value \\
\hline impact force & 1 & 71.4 & $\mathbf{2 . 6 8 E - 1 2}$ \\
impact duration & 1 & 8.9 & $\mathbf{3 . 9 8 E - 0 3}$ \\
pulling duration & 1 & 4.8 & $\mathbf{0 . 0 3}$ \\
contact area & 1 & 23.1 & $\mathbf{8 . 6 4 E - 0 6}$ \\
relative mucus coverage & 1 & 2.6 & 0.11 \\
specimen ID & 3 & 10.0 & $\mathbf{1 . 3 9 E - 0 5}$ \\
consecutive trial number & 1 & 0.5 & 0.49 \\
residuals & 70 & & \\
\end{tabular}

Response variable: adhesive strength $\log \left(\sigma_{\mathrm{adh}}\right)$

\begin{tabular}{lccc}
\hline Independent variable & DF & F value & p value \\
\hline contact pressure log $\left(\sigma_{\text {con }}\right)$ & 1 & 23.4 & $\mathbf{7 . 3 4 E - 0 6}$ \\
impact duration & 1 & 3.7 & 0.06 \\
pulling duration & 1 & 29.2 & $\mathbf{8 . 2 6 E - 0 7}$ \\
relative mucus coverage & 1 & 10.1 & $\mathbf{2 . 2 1 E - 0 3}$ \\
specimen ID & 3 & 3.8 & $\mathbf{0 . 0 1}$ \\
consecutive trial number & 1 & 0.2 & 0.65 \\
residuals & 71 & & \\
\end{tabular}

Response variable: relative mucus coverage $A_{\text {rel }}$

\begin{tabular}{lccc}
\hline Independent variable & DF & F value & p value \\
\hline contact pressure $\log \left(\sigma_{\text {con }}\right)$ & 1 & 3.1 & 0.08 \\
adhesive strength $\log \left(\sigma_{\text {adh }}\right)$ & 1 & 18.6 & $\mathbf{5 . 1 6 E - 0 5}$ \\
impact duration & 1 & 4.0 & $\mathbf{0 . 0 5}$ \\
pulling duration & 1 & 76.9 & $\mathbf{7 . 0 3 E - 1 3}$ \\
contact area & 1 & 0.1 & 0.78 \\
specimen ID & 3 & 5.7 & $\mathbf{1 . 4 7 E - 0 3}$ \\
consecutive trial number & 1 & 10.1 & $\mathbf{2 . 2 0 E - 0 3}$ \\
residuals & 70 & &
\end{tabular}

Response variable: impulse $J_{\text {adh }}$

\begin{tabular}{lrcc}
\hline Independent variable & DF & F value & p value \\
\hline impact force & 1 & 0.1 & 0.75 \\
impact duration & 1 & 0.8 & 0.37 \\
contact area & 1 & 74.4 & $\mathbf{1 . 1 5 E - 1 2}$ \\
relative mucus coverage & 1 & 4.2 & $\mathbf{0 . 0 4}$ \\
specimen ID & 3 & 2.1 & 0.11 \\
consecutive trial number & 1 & 2.1 & 0.15 \\
residuals & 71 & & \\
\hline
\end{tabular}


Table 2 | Estimated slopes resulting from the multivariate linear regression models

Response variable: adhesive force $F_{\text {adh }}$

\begin{tabular}{lrrrr}
\hline Independent variable & estimated slope & std. error & t value & p value \\
\hline impact force & 0.05 & 0.04 & 1.27 & 0.21 \\
impact duration & -1.95 & 0.87 & -2.25 & $\mathbf{0 . 0 3}$ \\
pulling duration & -0.02 & 0.03 & -0.85 & 0.40 \\
contact area & 0.13 & 0.29 & 0.45 & 0.66 \\
relative mucus coverage & -105.31 & 74.68 & -1.41 & 0.16 \\
consecutive trial number & -10.91 & 15.82 & -0.69 & 0.49 \\
intercept & 715.63 & 123.02 & 5.82 & $\mathbf{1 . 6 4 E - 0 7}$
\end{tabular}

intercept

715.63

123.02

$1.64 E-07$

Response variable: adhesive strength $\log \left(\sigma_{\mathrm{adh}}\right)$

\begin{tabular}{|c|c|c|c|c|}
\hline Independent variable & estimated slope & std. error & t value & $p$ value \\
\hline
\end{tabular}

Response variable: relative mucus coverage $A_{\text {rel }}$

\begin{tabular}{|c|c|c|c|c|}
\hline Independent variable & estimated slope & std. error & t value & p value \\
\hline
\end{tabular}

Response variable: impulse $J_{\text {adh }}$

\begin{tabular}{lcccc}
\hline Independent variable & estimated slope & std. error & t value \\
\hline impact force & $-3.80 \mathrm{E}-05$ & $2.73 \mathrm{E}-05$ & -1.4 & 0.17 \\
impact duration & $-7.12 \mathrm{E}-04$ & $5.72 \mathrm{E}-04$ & -1.25 & 0.22 \\
contact area & $6.73 \mathrm{E}-04$ & $1.89 \mathrm{E}-04$ & 3.56 & $\mathbf{6 . 5 8 E}-\mathbf{0 4}$ \\
relative mucus coverage & $7.61 \mathrm{E}-03$ & 0.05 & 0.16 & 0.87 \\
consecutive trial number & 0.02 & 0.01 & 1.45 & 0.15 \\
intercept & 0.18 & 0.08 & 2.11 \\
\hline
\end{tabular}

dance of relative mucus coverage on contact pressure, adhesive strength, impact and pulling duration, contact area, specimen ID, and consecutive trial number ( $\mathrm{p}=7.03 \mathrm{E}-013 ; \mathrm{F}=14.44$ on 9 and 70 degrees of freedom; adjusted $\mathrm{r}^{2}=0.60$ ). The residuals of the regression for relative mucus coverage were normally distributed $(\mathrm{p}=0.94$; $\mathrm{W}=0.99$ ). The relative mucus coverage differed significantly between the specimens (ANOVA, $\mathrm{p}=9.43 \mathrm{E}-04 ; \mathrm{F}=6.05$ on 3 and 76 degrees of freedom; Fig. 4).

Impulse. We evaluated the dependance of the impulse for the pulling phase (i.e. the sum of pulling forces during the entire duration of the pulling phase) on the force during the tongue impact, the duration of the tongue impact, the size of the contact area, the relative mucus coverage, the ID of the specimen, and the number of the consecutive trial during one session of force measurements. We found that significant amounts of the observed variation in the impulse are explained by differences in the contact area and the relative mucus coverage between experimental trials (Table 1). The size of the contact area is clearly positively correlated with the impulse of the adhesive event - a larger contact area results in higher forces that are maintained for longer time spans (Table 2). The multivariate linear regression model for the impulse explains overall significant amounts of the variation in our dataset $(\mathrm{p}=6.10 \mathrm{E}-10 ; \mathrm{F}=10.98$ on 8 and 71 degrees of freedom; adjusted $\left.r^{2}=0.50\right)$ and the residuals were normally distributed $(\mathrm{p}=0.43$; $\mathrm{W}=0.98)$. The adhesive impulse measured, differed significantly between specimens (ANOVA, $p=5.04 \mathrm{E}-08 ; \mathrm{F}=15.66$ on 3 and 76 degrees of freedom; Fig. 4).

\section{Discussion}

Our results demonstrate that frog tongue adhesive forces can be beyond the body weight of the animals and clearly outweigh any potential prey item. By using a flat glass surface for our experimental setup, we were able for the first time to investigate the relationship between the contact area and adhesive force of frog tongues and to calculate adhesive strength of this biological wet adhesive system. As expected, we found a positive correlation between contact pressure and adhesive strength, which suggests that the frog tongue can be considered as biological pressure sensitive adhesive system. Further, we found that less mucus coverage of the contact area is related to stronger adhesion, which might suggest that beyond the presence of mucus, other mechanisms add to the adhesiveness of frog tongues. Additionally, mucus-covered area correlates with the duration of the contact. The thickness of the mucus layer will have an influence on capillary and viscous forces and might also influence the frictional properties of the system. We have not measured the actual thickness of the mucus layer herein but we estimate the amount of mucus used based on the coverage of the contact area by residual mucus after the feeding strike. The total force with that the tongue of Ceratophrys sp. adheres to a prey object increases with shorter 

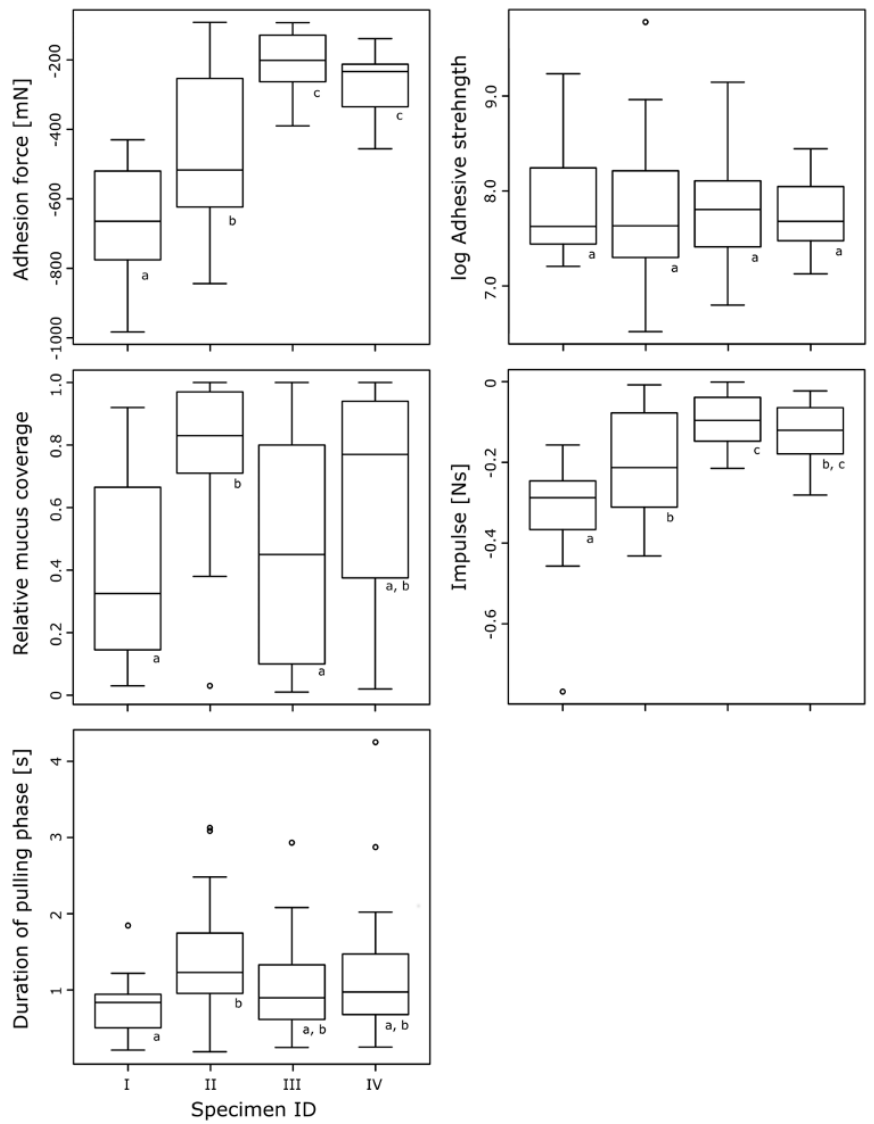

Figure $4 \mid$ Box-and-Whisker plots for functional variables discussed herein compared between specimens; different letters show statistical significance $(\mathrm{p}<0.05)$.

impact durations. This relationship between adhesion and impact duration can be explained by the dominant effect of viscous forces at thinner layers of mucus. Besides being able to catch elusive prey items, fast tongue projection mechanisms may also have evolved to generate a high contact pressure during the capture of heavy prey objects in amphibians.

Amphibian tongue forces have previously only been directly measured for the salamander Bolitoglossa occidentalis (Caudata: Plethodontidae) during impact of the tongue ${ }^{36}$ : salamanders with a body weight of 1.1-1.6 g generated maximum impact forces only equivalent to $0.04 \mathrm{~g}$. This results in a force to body weight ratio of less than 0.04 for B. occidentalis. Both, the impact and adhesive forces we measured herein for frogs in the genus Ceratophrys were consistently in the range of the body weight of the frogs and well beyond (impact up to 6.5 times the body weight; adhesion up to 3.4 times the body weight). In salamanders, tongue projection relies strongly on the tongue skeleton, which is fired out of the mouth ${ }^{6,37}$, while in frogs that use tongue projection, the muscular tongue is fired out of the mouth by a rapid depression of the lower jaw ${ }^{7,8}$; tongue projection in frogs and salamanders is considered to have evolved independently ${ }^{38,39}$.

Although we found mucus coverage to be variable, frog tongues are certainly not dry and thus provide an example of a biological wet adhesion system. Wet adhesion can occur due to effects of capillarity and viscosity (Stefan adhesion $)^{40}$. Also van der Waals interactions, suction, and glue effects may additionally contribute to adhesion of some biological wet adhesive system. We show that the impulse on the glass slide towards the direction of the frog, i.e. the force over the entire duration of the pulling event, is clearly positively correlated to the contact area. This suggests that a larger contact area allows for higher energy dissipation during contact breakage, which means that the adhesive bond can be maintained for prolonged periods of time. This is also the case in pressure sensitive adhesives (PSAs) ${ }^{41}$. During prey capture in frogs, this effect could be even more important than the strength of the adhesive bond because it will help to secure mobile prey while it is pulled into the mouth.

Interestingly, adhesive force per area (i.e. adhesive strength herein) was significantly higher in trials with low mucus coverage. This result demonstrates that mucus itself may not add simply as some sort of liquid adhesive or glue. Very likely, the microstructure and surface energy of the tongue surface as well as the surface properties of the prey item will have an important contribution to frog tongue adhesion. The positive correlation between the relative mucus coverage and the pulling duration in our experiment suggests that mucus might be spread into the contact area after the contact is made. This in turn suggests for natural occurring feeding events in Ceratophrys sp., that the mucus coverage of the contact area will be rather low because in our experimental setup, the frog tongues adhered much longer to the target (i.e. the glass slides in our experiment) than in regular feeding attempts because the glass slides were tightly attached to the force transducer and could not be swallowed by the frog. During regular feeding, the prey is pulled back almost immediately after the contact was made. Highspeed video recordings (Supplementary video file 2) suggest that in Ceratophrys sp., the tongue retraction movement starts only approximately $20 \mathrm{~ms}$ after the initial contact was made. These extremely short time intervals to establish a tight connection are also confirmed by previous studies on different anuran species $^{7,8,31-33}$. The EMG study by Gans and Gorniak ${ }^{15}$ found that tongue retraction actually is initiated up to $10 \mathrm{~ms}$ before the initial contact is made.

The adhesive strength also depends on the contact pressure (i.e. impact force per contact area) that the frog tongue applies to the opposing surface. Thus the tongue of Ceratophrys sp. provides a different adhesive mechanism than previously described in biological adhesion systems that rely on hair-like integumental outgrowths, like the attachment structures in geckoes ${ }^{42-46}$, insects ${ }^{4-52}$, or spiders ${ }^{53,54}$. In all of these systems, adhesion is initiated by shearing forces parallel to the opposing surface that aid in the proper alignment of the spatula-shaped setal tips on the surface (see also Filippov et al. ${ }^{55}$ ). In Ceratophrys, however, the tongue is pushed perpendicular to the surface of the prey item and not moved parallel to the opposing surface. A similar adhesion mechanism is known from the smooth adhesive pads on the legs of grass-hopers ${ }^{56}$, tree frogs ${ }^{57}$, and the stickcapture-apparatus in beetles of the genus Stenus ${ }^{58}$. Shearing forces on the level of the entire tongue are expected to be low but because the tongue will deform during impact and detachment along the glass surface, local shearing effects might be present but could not be detected with the one-dimensional setup of our force-measurement experiment.

The tongue of Ceratophrys sp. might be compared to pressuresensitive adhesives (PSAs), that are of common technical use e.g. in the form of adhesive tapes or labels ${ }^{41}$. The force over time relationship during detachment of the tongue in C. sp. (Fig. 2G) is very similar to those of PSAs ${ }^{41,59,60}$. In both, a high first adhesive peak is followed by a plateau where the adhesive force remains more or less constant over a longer period of time before the contact breaks. For PSAs, it has been shown that cavities arise during the peak force phase ${ }^{60}$. At some point these cavities will coalescence and thus cause the contact to break. However, depending on the properties of the PSA and the opposing surface, before coalescence, cavity formation may result in fibrillation ${ }^{59}$. PSA contacts with a high degree of fibrillation are characterized by a longer adhesion duration but also by the remains of PSA material (fibrils) on the contact material ${ }^{41}$. We observed fibrillation events in contact between the tongue of the frog and the 
glass slides during high-speed video recordings (Supplementary video file 1).

The comparison of frog tongues with PSAs actually might further explain the observed correlation between relative mucus coverage and adhesion duration in our experiment. Similar to PSAs, we found more mucus remains on the glass surfaces after trials in which the frogs tongues were attached for a longer period of time. Both, adhesion duration and mucus coverage, might be related to a higher degree of fibrillation during detachment, i.e. the contact was maintained for a longer time because of mucus fibrils between the tongue and the glass surface. However, in PSAs, higher fibrillation is related to better adhesive performance because the coalescence of cavities is prevented ${ }^{41}$. Our discovery of higher adhesive strength in trials with low mucus coverage and shorter adhesion duration would not have been predicted from this. Maybe we observed an opposite effect here: in feeding trials in which the frogs applied a higher pulling force during tongue retraction, contact duration was significantly shorter, also the mucus coverage decreased. Thus, higher adhesive strength may not be a consequence of low mucus coverage but vice versa, low mucus coverage results from higher stresses during the retraction phase and thus shorter adhesion durations. Individual differences between the specimens showed a similar pattern; e.g. the frog with ID II had a significantly prolonged pulling phase combined with a higher relative mucus coverage, and lower adhesive force compared to the similar sized frog with ID I (Fig. 4). It will be necessary to study the dynamics of tongue attachment and detachment in frogs with a focus on the contact area in future studies to clarify the adhesive mechanism for tongue feeding in more detail.

The adhesive strength we measured for the tongue of Ceratophrys sp. herein (max. $17 \mathrm{kPa}$ with $4 \mathrm{kPa}$ on average) is notably lower than e.g. in geckos that were reported to adhere with approximately $60 \mathrm{kPa}^{61}$. In another wet adhesive system that was described recently, the suctorial disk of the northern clingfish Gobiesox maeandricus, adhesive strengths of approximately $40 \mathrm{kPa}$ have been reported ${ }^{62}$. The toe pads of some tree frogs, however, show lower adhesive strength than the tongue of C. sp.; Barnes et al. ${ }^{57}$ measured approximately $1 \mathrm{kPa}$. Each of these systems is tuned to very different demands of performance. Tree frog and gecko toes are used for arboreal locomotion; the clingfish suctorial disc allows for strong quasi-static adhesion on rough surfaces submerged in seawater. In frog tongues, we have a very dynamic system in which high velocities and accelerations play a key role. Further, quantitative comparisons between the different biological adhesive systems are obscured by differences in the experimental design, like e.g. non-standardized surfaces for attachment and different approaches resulting in different failure modes at detachment of the tested systems.

Frogs within the Ceratophryidae are known to be generalist predators that can consume very large prey items relative to their own body dimension ${ }^{22,29}$. Although the adhesive strengths we measured herein are weaker than in other biological adhesive systems, from a biological point of view, it will be very unlikely that Ceratophrys sp. will have a need to lift prey that is more than its own body weight with the tongue. It remains to be tested, however, if frog tongues achieve similar adhesive strengths on surfaces with variable structures and surface chemistries that more closely resemble the properties of natural prey surface materials (e.g. fur, feathers, cuticle) than in our experiment herein. The combination of highly dynamic tongue projection, high adhesive strength, and potentially the versatility to attach to structurally and chemically variable surfaces (e.g. fur, feathers, cuticle), makes frog tongues a unique example for biological wet adhesion.

\section{Methods}

Specimens and housing. The experiment herein was approved by the animal welfare representative of the Christian-Albrechts-Universität Kiel and the Ministry of agriculture, the environment, and rural areas of the federal state Schleswig-Holstein, Germany (file number V 312-72241.121-29); all experimental trials reported herein were carried out in accordance with the approved guidelines. The force measurement experiments herein were performed with four specimens of Ceratophrys sp. This frog species is a fossorial sit-and-wait predator native to Argentina, Bolivia, Brazil, and Paraquay (AmphibiaWeb.org, 2013). The specimens used herein were captive breed and purchased from the local pet trade. The four individuals comprised two adults (ID I: $63 \mathrm{~mm}$ snout-vent-length (SVL) and $63.1 \mathrm{~g}$ body weight; ID II: $70 \mathrm{~mm} \mathrm{SVL}$ and $72.7 \mathrm{~g}$ body weight) and two juveniles (ID III: $28 \mathrm{~mm}$ SVL and $12.7 \mathrm{~g}$ body weight; ID IV: $31 \mathrm{~mm}$ SVL and $12.7 \mathrm{~g}$ body weight). The two juveniles were identified as Ceratophrys cranwelli, the two adult frogs represent the so-called 'fantasy' colormorph, which does not occur in the wild and is bred by crossing C. cranwelli with $C$. cornuta. The animals were individually housed at temperatures of $29^{\circ} \mathrm{C}$ during the day and $26^{\circ} \mathrm{C}$ at night. The terrariums in which the frogs were housed were filled with loose substrate to a depth of approximately $5 \mathrm{~cm}$ in which the frogs could bury themselves. Usually the frogs were found to be half buried and only occasionally they moved to a different location. We moistened the terrariums daily to sustain a relative humidity of approximately 70 to $80 \%$. Before the onset of the feeding experiment, the animals were fed twice a week an alternating diet of crickets (Gryllus bimaculatus), grasshoppers (Schistocerca gregaria), wax-worms (Zophobas morio), and earthworms (Lumbricus terrestris).

Experimental setup. We performed force measurements during the normal feeding routine of the frogs twice a week. During each experimental session, a maximum of five trials per individual was recorded. Overall, we measured the forces during twenty feeding trials for each frog, i.e. a total of 80 feeding events.

For force measurements, we used a MP 100 data acquisition system (BIOPAC Systems Inc., Goleta, CA, USA) that was equipped with two types of force transducers (World Precision Instruments, Sarasota, FL, USA): (1) a $100 \mathrm{~g}$ force transducer for measurements with the juvenile frogs; (2) a 1,000 $\mathrm{g}$ force transducer for measurements with the two adult specimens. The MP 100 data acquisition system was connected to a notebook PC and controlled by the software AcqKnowledge 3.7. The data acquisition rate was set to $1,000 \mathrm{~Hz}$. We directly attached a microscope glass slide to the force transducer by using a stripe of two sided adhesive tape (tesa ${ }^{\circledR}$ Double-Sided Tape Universal, tesa SE, Hamburg, Germany) (Fig. 1). For calibration of the force transducers with the attached glass slides, we used a $20 \mathrm{~g}$ measuring weight for the $100 \mathrm{~g}$ force transducer, respectively a $100 \mathrm{~g}$ measuring weight for the $1,000 \mathrm{~g}$ force transducer. We moved the force transducers into a horizontal position and placed the measuring weights centrally onto the attached glass slides. The area of the measuring weights was $69.1 \mathrm{~mm}^{2}(20 \mathrm{~g})$ and $103.7 \mathrm{~mm}^{2}(100 \mathrm{~g})$, which was in the same range as the frog tongue areas during the experimental trials (tongue contact area for each feeding trial is presented in Supplementary Table 1). For the experiment, the force transducer was then placed in a vertical position. Because we calibrated the force measurement setup horizontally, we measured an offset force in vertical direction. To account for this, we recorded these offset forces for $10 \mathrm{~s}$ prior to the actual force measurement and used the mean offset force to tare our system. After each force measurement, we exchanged the glass slides and repeated the calibration procedure.

We placed the vertically oriented force transducers with the attached glass slides into the terrariums of the frogs by using a laboratory stand, clamps, and rods. Vertically, we placed the glass slide immediately above the ground; horizontally, we centered the glass slide approximately $2 \mathrm{~cm}$ in front of the rostral tip of the nose of the frogs (Figs. 1,2A). Generally, the frogs did not move and remained half buried during this procedure. We then placed a cricket immediately behind the glass slide and held it in place with a forceps. As the frogs tried to capture the cricket, the impact and pulling forces of the tongue acting on the glass slide were recorded over time until the frog tongue became detached (Fig. 2A-F). It was possible for the frogs to lunge towards the force transducer and thus to shorten the distance of tongue projection. To avoid a negative learning effect for the frogs, we moved the experimental setup out of the terrariums after each trial and the frogs were allowed to feed the cricket before the next force measurement trial.

We were able to control the region where the tongue impacted the glass slide by placing the cricket centrally behind the force transducer. However, we estimated the accuracy of the recorded forces in case the frog tongue hits the glass slide and thus the force transducer with an offset from the center. For this, we moved the force transducers with the attached glass slide in a horizontal position and placed a measuring weight (20 g for the $100 \mathrm{~g}$ force transducer and $100 \mathrm{~g}$ for the $1,000 \mathrm{~g}$ force transducer) centrally and with an offset of $1.0,2.0$, respectively $3.0 \mathrm{~cm}$ off the center onto the glass slide and recorded the force acting on the force transducer. We found a maximum deviation between the actual weight force of the measuring weights of $10.2 \%$ for the $100 \mathrm{~g}$ force transducer and $4.9 \%$ for the $1,000 \mathrm{~g}$ force transducer (Supplementary table 2).

Because the glass slide was placed vertically in front of the frogs, the tongues did not reach their full extension during our experimental trials. In chameleons $s^{2,20,63}$, Salamanders ${ }^{11}$, and the tomato frog Dyscophus guinet $i^{64}$ it was demonstrated that tongues decelerate before the impact with the prey along their projected trajectories. Thus, in our experimental setup, we might overestimate the forces with which the tongue impacts on a prey item by shortening the tongue projection distance. However, besides the absolute values reported herein for the tongue impact forces, this overestimation is supposed to have no effect on the correlations between tongue feeding parameters discussed herein. Further, video evidence suggests that, during regular feeding in Ceratophrys sp., the tongue is also not always fully projected depending on the distance to the prey (Supplementary movie 3). 
Data analysis. The raw force measurement data were imported into the R computing environment version 2.14.2 (R-Foundation for statistical computing, http://www.rproject.org). Figure $2 \mathrm{G}$ shows a typical force over time relationship during one experimental trial. We then extracted the following parameters: $F_{i m p}$ - the maximal force during impact of the tongue, $t_{i m p}$ - the duration of the impact (i.e. the width of the impact peak, Fig. $2 \mathrm{G}), F_{a d h}$ - the maximal force during tongue adhesion, and $t_{\text {pull }}$ the duration of the pulling phase until the tongue was detached. Further we calculated the adhesive impulse $J_{a d h}$ :

$$
J_{a d h}=\int F_{a d h} * d t
$$

For this purpose, we estimated the area under the force-time curve for the adhesive phase (Fig. 2G) by calculating:

$$
J_{\text {adh }}=\left(\Sigma F_{a d h}\right) * t_{\text {pull }}
$$

After each force measurement trial, we studied the area covered by remains of mucus on the microscope glass slides, i.e. the prints of the tongue (Fig. 3). We digitally photographed the tongue prints on $1 \mathrm{~mm} \times 1 \mathrm{~mm}$ scale paper. We then measured the total area $A$ of the tongue prints with the image analyzation software Image J 1.47 (avaialbe at http://rsbweb.nih.gov/ij/download.html). In several trials, the inside of the tongue print was not entirely covered by mucus (Fig. 3). To estimate the degree of mucus coverage, we also measured the mucus free area $A_{\text {free }}$ and calculated relative mucus coverage $A_{\text {rel }}$ as:

$$
A_{\text {rel }}=\left(A-A_{\text {free }}\right) / A
$$

Further, we calculated both, the contact pressure $\sigma_{c o n}$ and adhesive strength $\sigma_{a d h}$ as:

$$
\sigma_{c o n}=F_{i m p} / A
$$

$$
\sigma_{a d h}=F_{a d h} / A
$$

We tested the variables evaluated herein for normal distribution by performing a Shapiro-Wilk-test; normal distribution was assumed if the null-hypothesis of normal distribution was accepted ( $p>0.05$ ). Most of the variables herein are not normally distributed over the entire dataset but follow a normal distribution within measurements for a single specimen (Supplementary Table 3). For the contact pressure $\sigma_{c o n}$ and adhesive strength $\sigma_{a d h}$, the data was transformed to follow a normal distribution by calculating the $\log _{10}$.

For statistical analysis of our data, we performed a multivariate linear regression analysis with $\mathrm{R}$ 2.14.2. We evaluated the contribution of single parameters to our multivariate linear models by analyzing the ANOVA table for our multivariate regression results - the level of significance was set to $\mathrm{p}<0.05$. We tested four parameters: (1) the adhesive force $F_{a d h}$ as dependent variable with the impact force $F_{i m p}$, the duration of the impact $t_{i m p}$, the duration of the pulling phase $t_{p u l l}$, the total contact area A, the relative mucus coverage $A_{\text {rel }}$, the specimen ID, and the number of the trial on a given day as independent variables; (2) adhesive strength $\sigma_{a d h}$ as dependent variable with the contact pressure $\sigma_{c o n}$, the duration of the impact $t_{i m p}$, the duration of the pulling phase $t_{\text {pull }}$, the relative mucus coverage $A_{r e l}$, the specimen ID, and the number of the trial as independent variables; (3) relative mucus coverage $A_{\text {rel }}$ as dependent variable with the duration of the pulling phase $t_{\text {pull }}$, the duration of the impact $t_{i m p}$, the contact pressure $\sigma_{c o n}$, the adhesive strength $\sigma_{a d h}$, the contact area A, the specimen ID, and the trial number per day as independent variables; (4) impulse $J_{a d h}$ in dependance on the contact area $A$, the relative area covered by mucus $A_{\text {rel }}$, the impact force $F_{i m p}$, the duration of the impact $t_{i m p}$, the specimen ID, and the trial number. Because several of the variables for the multivariate linear regression did not follow a normal distribution, we evaluated the robustness of our regression models by testing the residuals of the multivariate linear regression results for normal distribution with a Shapiro-Wilk test. To evaluate individual differences between specimens, we further calculated one-way ANOVAs for adhesive force $F_{a d h}$, adhesive strength $\sigma_{a d h}$, relative mucus coverage $A_{r e l}$, impulse $J_{a d h}$, and the duration of the pulling phase $t_{p u l l}$ as dependant on specimen ID. Based on the ANOVAs, we performed a Tukey's honest significant difference (HSD) test in which we pair-wise compared individuals for statistical significance $(\mathrm{p}<0.05)$.

1. Iwasaki, S. Evolution of the structure and function of the vertebrate tongue. J Anat 201, 1-13 (2002)

2. Anderson, C. V. \& Deban, S. M. Ballistic tongue projection in chameleons maintains high performance at low temperature. Proc Natl Acad Sci U S A 107, 5495-5499 (2010).

3. Anderson, C. V., Sheridan, T. \& Deban, S. M. Scaling of the ballistic tongue apparatus in chameleons. J Morphol 273, 1214-1226 (2012).

4. Wainwright, P. C. \& Bennett, A. F. The mechanism of tongue projection in chameleons: II. Role of shape change in a muscular hydrostat. J Exp Biol 168, 23-40 (1992).

5. Wainwright, P. C. \& Bennett, A. F. The mechanism of tongue projection in chameleons: I. Electromyographic tests of functional hypotheses. J Exp Biol 168, $1-21$ (1992).
6. Deban, S. M., Wake, D. B. \& Roth, G. Salamander with a ballistic tongue. Nature 389, 27-28 (1997).

7. Nishikawa, K. C. Feeding in frogs. in Feeding: Form, function, and evolution in tetrapod vertebrates (ed. Schwenk, K.) 117-147 (Academic Press, 2000).

8. Nishikawa, K. C. \& Gans, C. Mechanisms of tongue protraction and narial closure in the marine toad Bufo marinus. J Exp Biol 199, 2511-2529 (1996).

9. Nishikawa, K. C., Deban, S. M., Anderson, C. W. \& O'Reilly, J. C. The evolution of neural circuits controlling feeding behavior in frogs. Brain Behav Evol 40, 125-140 (1992).

10. Schwenk, K. \& Rubega, M. A. Diversity of vertebrate feeding systems. in Physiological and ecological adaptations to feeding in vertebrates (eds. Starck, M. J. \& Wang, T.) 1-41 (Science Publishers, 2005).

11. Deban, S. M., O’Reilly, J. C., Dicke, U. \& van Leeuwen, J. L. Extremely high-power tongue projection in plethodontid salamanders. J Exp Biol 210, 655-667 (2007).

12. de Groot, J. H. \& van Leeuwen, J. L. Evidence for an elastic projection mechanism in the chameleon tongue. Proc Biol Sci 271, 761-770 (2004).

13. Regal, P. J. \& Gans, C. Functional aspects of the evolution of frog tongues. Evolution 30, 718-734 (1976).

14. Gans, C. \& Gorniak, G. C. How does the toad flip its tongue? Test of two hypotheses. Science 216, 1335-1337 (1982).

15. Gans, C. \& Gorniak, G. C. Functional morphology of lingual protrusion in marine toads (Bufo marinus). Am J Anat 163, 195-222 (1982).

16. Deban, S. M. Constraint and convergence in the evolution of salamander feeding. in Vertebrate biomechanics and evolution (ed. Bels, V. L., Gase, J.-P. \& Casinos, A.) 161-178 (Bios Scientific Publishers, 2003).

17. Herrel, A., Deban, S. M., Schaerlaeken, V., Timmermans, J.-P. \& Adriaens, D. Are morphological specializations of the hyolingual system in chameleons and salamanders tuned to demands on performance? Physiol Biochem Zool 82, 29-39 (2009).

18. Herrel, A., Meyers, J. J., Nishikawa, K. C. \& De Vree, F. Morphology and histochemistry of the hyolingual apparatus in chameleons. J Morphol 249, 154-170 (2001).

19. Herrel, A., Meyers, J. J., Timmermans, J.-P. \& Nishikawa, K. C. Supercontracting muscle: producing tension over extreme muscle lengths. J Exp Biol 205, 2167-2173 (2002).

20. Herrel, A., Meyers, J. J., Aerts, P. \& Nishikawa, K. C. The mechanics of prey prehension in chameleons. J Exp Biol 203, 3255-3263 (2000).

21. Branch, W. R. Two exceptional food records for the african bullfrog, Pyxicephalus adspersus (Amphibia, Anura, Ranidae). J Herpetol 10, 266-268 (1976).

22. Chávez, G., Venegas, P. J. \& Lescano, A. Two new records in the diet of Ceratophrys cornuta Linneaus, 1758 (Anura: Ceratophrydae). Herpetol Notes 4, 285-286 (2011).

23. Duellman, W. E. \& Trueb, L. Biology of amphibians. (John Hopkins University Press, 1994).

24. Figueiredo-de-Andrade, C. A., Caram, Joana \& De Carvalho-E-Silva, S. P. Frog eats frog: report of three cases from the Atlantic rain forest, southeastern Brazil. Salamandra 48, 230-232 (2012).

25. McLeod, D. S. Limnonectes megastomias (Big-mouthed Frog). Diet and Ornithophagy. Herpetol Rev 40, 205-206 (2009).

26. Lynch, J. D. Evolutionary relationships, osteology, and zoogeography of leptodactyloid frogs. Univ Kansas Mus Nat Hist Misc publ 53, 1-238 (1971).

27. Wild, E. R. Description of the adult skeleton and developmental osteology of the hyperossified horned frog, Ceratophrys cornuta (Anura: Leptodactylidae). J Morphol (1997).

28. Lynch, J. D. Relationships of the frogs of the genus Ceratophrys (Leptodactylidae) and their bearing on hypotheses of pleistocene forest refugia in South-America and punctuated equilibria. Syst Zool 31, 166-179 (1982).

29. Duellman, W. E. \& Lizana, M. Biology of a sit-and-wait predator, the leptodactylid frog Ceratophrys cornuta. Herpetologica 50, 51-64 (1994).

30. Monroy, J. A. \& Nishikawa, K. C. Prey capture in frogs: alternative strategies, biomechanical trade-offs, and hierarchical decision making. J Exp Zool A Ecol Genet Physiol 315A, 61-71 (2011).

31. Deban, S. M. \& Lappin, A. K. Thermal effects on the dynamics and motor control of ballistic prey capture in toads: maintaining high performance at low temperature. J Exp Biol 214, 1333-1346 (2011).

32. Deban, S. M. \& Nishikawa, K. C. The kinematics of prey capture and the mechanism of tongue protraction in the Green Tree Frog Hyla cinerea. J Exp Biol 170, 235-256 (1992).

33. Nishikawa, K. C. \& Roth, G. The mechanism of tongue protraction during prey capture in the frog Discoglossus pictus. J Exp Biol 159, 217-234 (1991).

34. Iwasaki, S., Iwabuchi, Y. \& Okumura, Y. Histological and ultrastructural studies of the effects of tachykinins on protein secretion from the lingual epithelium and the lingual gland of the Tokyo daruma pond frog (Rana porosa porosa). Arch Oral Biol 43, 463-471 (1998).

35. Zylberberg, L. Histochemistry and ultrastructure of amphibian lingual glands and phylogenetic relations. Histochem J 9, 505-520 (1977).

36. Thexton, A. J., Wake, D. B. \& Wake, M. H. Tongue function in the salamander Bolitoglossa occidentalis. Arch Oral Biol 22, 361-366 (1977).

37. Lombard, R. E. \& Wake, D. B. Tongue evolution in the lungless salamanders, family plethodontidae. I. Introduction, theory and a general model of dynamics. J. Morphol. 148, 265-286 (1976). 
38. Bramble, D. M. \& Wake, D. B. Feeding mechanisms of lower tetrapods. in Functional vertebrate morphology (eds. Hildebrand, M., Bramble, D. M., Liem, K. F. \& Wake, D. B.) 230-261 (Cambridge: Harvard University Press, 1985).

39. Deban, S. M., O'Reilly, J. \& Nishikawa, K. C. The evolution of the motor control of feeding in amphibians. in Am Zool 41, 1280-1298 (2001).

40. Emerson, S. B. \& Diehl, D. Toe pad morphology and mechanisms of sticking in frogs. Biol J Linn Soc 13, 199-216 (1980).

41. Creton, C. Pressure-Sensitive Adhesives: An Introductory Course. MRS Bull 28, 434-439 (2003).

42. Autumn, K. et al. Adhesive force of a single gecko foot-hair. Nature 405, 681-685 (2000).

43. Autumn, K. \& Gravish, N. Gecko adhesion: evolutionary nanotechnology. Phil Trans $R$ Soc A 366, 1575-1590 (2008).

44. Autumn, K. Properties, principles, and parameters of the gecko adhesive system. in Biological adhesives (eds. Smith, A. \& Callow, J.) 225-256 (Springer Berlin Heidelberg, 2006).

45. Tian, Y. et al. Adhesion and friction in gecko toe attachment and detachment. Proc Nat Acad Sci U S A 103, 19320-19325 (2006).

46. Hiller, U. Untersuchungen zum Feinbau und zur Funktion der Haftborsten von Reptilien. Zoomorphology 62, 307-362 (1968).

47. Beutel, R. G. \& Gorb, S. N. Ultrastructure of attachment specializations of hexapods (Arthropoda): evolutionary patterns inferred from a revised ordinal phylogeny. J Zool Syst Evol Res 39, 177-207 (2001).

48. Gorb, S. Biological microtribology: anisotropy in frictional forces of orthopteran attachment pads reflects the ultrastructure of a highly deformable material. $P$ Roy Soc B-Biol Sci 267, 1239-1244 (2000).

49. Gorb, S. N., Gorb, E. V. \& Kastner, V. Scale effects on the attachment pads and friction forces in syrphid flies (Diptera, Syrphidae). J Exp Biol 204, 1421-1431 (2001)

50. Gorb, S. N. et al. Structural design and biomechanics of friction-based releasable attachment devices in insects. Int Comp Biol 42, 1127-1139 (2002).

51. Niederegger, S. \& Gorb, S. N. Tarsal movements in flies during leg attachment and detachment on a smooth substrate. I Insect Physiol 49, 611-620 (2003).

52. Bullock, J. M. R., Drechsler, P. \& Federle, W. Comparison of smooth and hairy attachment pads in insects: friction, adhesion and mechanisms for directiondependence. J Exp Biol 211, 3333-3343 (2008).

53. Arzt, E., Gorb, S. N. \& Spolenak, R. From micro to nano contacts in biological attachment devices. Proc. Natl. Acad. Sci. U.S.A. 100, 10603-10606 (2003).

54. Wolff, J. O. \& Gorb, S. N. The influence of humidity on the attachment ability of the spider Philodromus dispar (Araneae, Philodromidae). P Roy Soc B-Biol Sci 279, 139-143 (2011)

55. Filippov, A., Popov, V. L. \& Gorb, S. N. Shear induced adhesion: Contact mechanics of biological spatula-like attachment devices. J Theor Biol $\mathbf{2 7 6}$ 126-131 (2011).

56. Jiao, Y., Gorb, S. N. \& Scherge, M. Adhesion measured on the attachment pads of Tettigonia viridissima (Orthoptera, insecta). J Exp Biol 203, 1887-1895 (2000).

57. Barnes, W. J. P., Oines, C. \& Smith, J. M. Whole animal measurements of shear and adhesive forces in adult tree frogs: insights into underlying mechanisms of adhesion obtained from studying the effects of size and scale. J Comp Physiol A 192, 1179-1191 (2006).
58. Koerner, L., Gorb, S. N. \& Betz, O. Adhesive performance of the stick-capture apparatus of rove beetles of the genus Stenus (Coleoptera, Staphylinidae) toward various surfaces. J Insect Physiol doi:10.1016/j.jinsphys.2011.11.001 (2011).

59. Zosel, A. Adhesive failure and deformation behaviour of polymers. J Adh (1989).

60. Lakrout, H., Creton, C. \& Sergot, P. Direct observation of cavitation and fibrillation in a probe tack experiment on model acrylic pressure-sensitiveadhesives. J Adh (1999).

61. Irschick, D. J., Herrel, A. \& Vanhooydonck, B. Whole-organism studies of adhesion in pad-bearing lizards: creative evolutionary solutions to functional problems. J Comp Physiol A 192, 1169-1177 (2006).

62. Wainwright, D. K., Kleinteich, T., Kleinteich, A., Gorb, S. N. \& Summers, A. P. Stick tight: suction adhesion on irregular surfaces in the northern clingfish. Biol Lett 9, 20130234-20130234 (2013).

63. Wainwright, P. C., Kraklau, D. M. \& Bennett, A. F. Kinematics of tongue projection in Chamaeleo oustaleti. J Exp Biol 159, 109-133 (1991).

64. Monroy, J. A. \& Nishikawa, K. C. Prey location, biomechanical constraints, and motor program choice during prey capture in the tomato frog, Dyscophus guineti. J Comp Physiol A 195, 843-852 (2009).

\section{Acknowledgments}

We wish to express our gratitude to the members of the functional morphology and biomechanics group at the Christian-Albrechts-Universität Kiel. Lars Heepe and Philipp $\mathrm{Bu} \beta$ hardt gave a helpful introduction into the data acquisition system. Further, we wish to thank Stephan Bootsmann for his support in the housing of the frog specimens, which were used in this study. Financial support for our ongoing research on the evolution of amphibian feeding systems was provided by the Volkswagen Foundation (grant I 84/206 to T.K.) and the German Research Foundation (grant KL-2707/2-1 to T.K.)

\section{Author contributions}

T.K. and S.N.G. designed the experiment, analyzed the data, and wrote this article. T.K. performed the experiment.

\section{Additional information}

Supplementary information accompanies this paper at http://www.nature.com/ scientificreports

Competing financial interests: The authors declare no competing financial interests.

How to cite this article: Kleinteich, T. \& Gorb, S.N. Tongue adhesion in the horned frog Ceratophrys sp. Sci. Rep. 4, 5225; DOI:10.1038/srep05225 (2014).

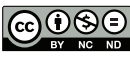

This work is licensed under a Creative Commons Attribution-NonCommercialNoDerivs 4.0 International License. The images or other third party material in this article are included in the article's Creative Commons license, unless indicated otherwise in the credit line; if the material is not included under the Creative Commons license, users will need to obtain permission from the license holder in order to reproduce the material. To view a copy of this license, visit http:// creativecommons.org/licenses/by-nc-nd/4.0/ 